\title{
Erste Annäherung
}

Unsere Vorlesung zur Romantik zwischen zwei Welten wendet sich folglich einem Themenbereich zu, der auf den ersten Blick relativ klar umrissen scheint. In einschlägigen Literaturgeschichten hat die Romantik ihren festen Platz: Autorinnen und Autoren, die zur Romantik gerechnet werden, kommt gerade auf der Ebene nationaler Literaturgeschichtsschreibung ein großes, bisweilen übermächtiges Prestige zu. Literarische Werke, die der Romantik zugeschlagen werden, sind vor allem in nationalphilologischen Darstellungen von größter Relevanz; zudem sind die entsprechenden Texte und Schriften nicht selten noch heute von ungeheurer Popularität. Womit hat dieser hohe Bekanntheitsgrad, dieses unbestreitbare Prestige der Literatur - oder eigentlich müsste ich sagen: der Literaturen - der Romantik zu tun?

Zweifellos liegt dies, wie wir noch sehen werden, unter anderem an den literarästhetisch herausragenden Texten und ihren Autorinnen und Autoren selbst, an spezifischen Epochenmentalitäten und vielen anderen Faktoren wie etwa der Tatsache, dass diese Literaturen zeitgleich entstanden zu Gründung und Aufbau der jeweiligen Nationalphilologien. Daher ist ein besonderer Grund zumindest in der Frage bereits implizit mitenthalten: Es liegt sicherlich auch an der jeweils stark forcierten nationalen Dimension dieser jeweiligen Literaturen. Gerade weil das Nationale - in Deutschland und Italien, aber zweifellos auch in vielen anderen Ländern, die sich wie Frankreich oder England, Argentinien oder Mexiko bereits als Nationalstaaten konstituiert hatten - einen nicht unwesentlichen Aspekt der romantischen Kunst- und Literaturproduktion ausmachte, war selbstverständlich eine spezifische Beziehung zwischen nationaler Literatur und nationaler Literaturgeschichtsschreibung gegeben. Vor allem aber auch ein Zusammenhang mit kollektiven, nationalen oder nationalistischen Identitätsbildungsprozessen.

All dies schlug sich auch in einer starken Kanonisierung von Kunst und Literatur der Romantik nieder. Dass die nur auf den ersten Blick erstaunliche Präsenz des Nationalismus in den europäischen Gesellschaften - und nicht allein in diesen: Die europäischen Kolonialismen waren auch ausgezeichnete Exporteure - ein anhaltendes Erbe der romantischen Periode ist, brauche ich Ihnen nicht zu sagen. Denn auch die Nationalismen des 19. Jahrhunderts geben uns deutliche Hinweise auf die Aktualität der Romantik unter den verschiedenartigsten gesellschaftlichen Bedingungen. Wir werden uns mit diesem fortbestehenden Erbe beschäftigen müssen und wollen es nicht verdrängen: Denn nicht nur Sigmund Freuds Theorien entnehmen wir, dass das einmal Verdrängte mit ungeheurer Wucht zurückzukehren sucht.

Aus all den genannten Aspekten zu schließen, dass Kunst und Literatur der Romantik eine jeweils nationale und auf die eigene Nation bezogene Kunst ist, 
wäre jedoch ein Kurzschluss, eine unsinnige Verkürzung. Denn gerade in diese Zeit fällt eine Vielzahl weltumspannender Entwicklungen, die mit der massiven Expansion Europas zusammenhängen. Denn das 19. Jahrhundert umfasst an seinem Beginn die Ausläufer der zweiten Phase beschleunigter Globalisierung, während es in seinem letzten Drittel bereits die dritte Phase dieser globalisierten Beschleunigung beinhaltet. ${ }^{1}$ Es ist also von grundlegenden Globalisierungsphasen gleichsam ,gerahmt' und es wäre grundfalsch, in ihm nur das Jahrhundert der Nationalismen oder nationalen Abschottungen zu sehen. Doch wir werden dies im weiteren Verlauf unserer Vorlesung noch mit aller Deutlichkeit anhand der groben geschichtlichen Züge ebenso wie an zahlreichen künstlerischen Details erkennen.

Wir stehen damit grundsätzlich vor einer bipolaren Struktur, die sich auf die gesamte Romantik - und dies wurde nur allzu oft nicht gesehen - wie auf die romantische Kunst und Literatur durchpaust: Es handelt sich um die Spannung zwischen Nation und Welt, Nationalstaat und Menschheit, Besinnung auf das Regionale sowie Lokale einerseits und Reflexion der weltumspannenden Entwicklungen andererseits. Diese Spannung zeigt sich auch in der noch zu besprechenden Herausbildung des Gegensatzes zwischen Nationalliteratur und Weltliteratur, der für unsere Sichtweise wie auch noch für gänzlich anders geartete Theoriebildungen des 21. Jahrhunderts wichtig ist.

Unsere Vorlesung wird versuchen - und ich denke, dass sie an diesem Punkt neue Wege beschreitet -, diese bipolare Struktur adäquat und auf den unterschiedlichsten Ebenen zu erfassen. Sie will daher Romantik in zwei Welten, Romantik in Europa und Amerika untersuchen sowie herausarbeiten, dass es jenseits nationalliterarischer Entwicklungen Prozesse anderer Art gab, die gerade von einer nationalliterarischen Literaturgeschichtsschreibung nicht erfasst werden konnten und vielleicht nicht erfasst werden sollten. Es geht also um die Romantik in zwei Welten und damit um die Romantik zwischen den Polen von Nationalem und Internationalem, Volk und Menschheit, Nationalliteratur und Weltliteratur, zwischen Nationalphilologie und - um mit Erich Auerbach ${ }^{2} \mathrm{zu}$ sprechen - Philologie der Weltliteratur.

1 Zur Theorie und den einzelnen Phasen beschleunigter Globalisierung vgl. Ette, Ottmar: TransArea. Eine literarische Globalisierungsgeschichte. Berlin - Boston: Walter de Gruyter 2012; Übersetzungen ins Englische (2016) und Französische (2019) liegen vor, eine Übersetzung ins Chinesische ist auf dem Weg.

2 Vgl. Auerbach, Erich: Philologie der Weltliteratur. In: Weltliteratur. Festgabe für Fritz Strich. Bern 1952, S. 39-50; wieder aufgenommen in Auerbach, Erich: Gesammelte Aufsätze zur romanischen Philologie. Herausgegeben von Fritz Schalk und Gustav Konrad. Bern - München: Francke Verlag 1967, S. 301-310. 
Bevor wir in der Folge zu anderen bipolaren Strukturen und offenen bipolaren Strukturierungen gelangen, die ebenfalls die Romantik in ihrem Spannungsgeflecht grundlegend prägen - also vor allem die Bipolarität von Individuum und Kollektivität oder Gemeinschaft einerseits sowie die Zweipoligkeit von Historisierung und Verräumlichung andererseits -, sollten wir uns um diese erstgenannte Zweipoligkeit bei unserer ersten Annäherung etwas genauer bemühen. Wie also wäre das Verhältnis zwischen dem Raum des Nationalen und dem Kosmos, dem Welten-Raum zu denken? Wie kann in diese Beziehung von Europa aus das Individuum hineingestellt werden, und mehr noch: Welche spezifischen Wissensbestände werden benötigt und entwickelt, um das neue Verhältnis von Nationalem und Internationalem, ja Transnationalem zu denken?

Wir können vor diesem Hintergrund die Romantik nicht begreifen, wenn wir nicht einen kurzen Blick werfen auf jene philosophische, literarische und mentalitätsgeschichtliche Epoche, die wir als Aufklärung, als „Enlightenment“, als „Siècle des Lumières“ zu bezeichnen pflegen. Dynamik und Wucht der Romantik sind nicht zu erfassen und zu begreifen, lässt man außer Acht, wogegen sie sich absetzt, womit sie ganz bewusst kontrastiert und wessen denkerische und philosophische Entwicklungen sie gleichwohl eine volle Drehung in der Spirale der Geschichte weiterdreht.

In einer seiner Anmerkungen zum Discours sur l'origine et les fondements de l'inégalité parmi les hommes äußerte sich Jean-Jacques Rousseau 1755 zum damaligen Stand anthropologischer Kenntnisse bei den Europäern. Er stellte dabei das Problem der asymmetrischen Beziehungen zwischen jenen Informationen, die von Reisenden aus unterschiedlichen europäischen Ländern stammten, und den Überlegungen und Theorien der Philosophen heraus, welche auf diesen Nachrichten und Kenntnissen basierten. Es ist, wie mir scheint, eine der grundlegenden Fragen zum Universalitätsanspruch europäischer Philosophie und dem verschwindend kleinen Kenntnisstand, auf dem diese Ansprüche beruhen, eine universalgültige Philosophie des Menschengeschlechts zu entwickeln. Lassen Sie mich sogleich hinzufügen, dass ich mich sehr wohl frage, ob sich an diesem Missverhältnis in unserer Zeit wirklich etwas Grundlegendes geändert hat. Es scheint vielmehr, als ob wir heute jenseits einer unbedingt notwendigen, aber vernachlässigten interkulturellen Philosophie in der deutschen, französischen, englischen oder italienischen Philosophie nach wie vor Konzepte entfalten, die universalistisch gedacht zu sein vorgeben, aber letztlich nur Beispiele eines Denkens in den Traditionslinien Englands, Italiens, Frankreichs oder Deutschlands sind. 


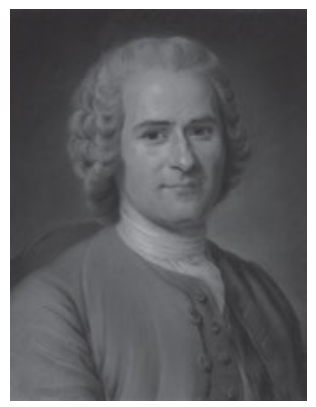

Abb. 1: Jean-Jacques Rousseau (Genf, 1712 - Ermenonville, 1778).

Jean-Jacques Rousseau betonte dabei die Wichtigkeit, mit einer derartigen Asymmetrie Schluss zu machen, die auf der seiner Ansicht nach fehlenden Vorbereitung der überwiegenden Mehrzahl der Reisenden beruhte. Zugleich aber hielt er einen gewissen Mangel hinsichtlich der Orientierung mancher „hommes éclairés“ fest, die sich vielfältigen Gefahren ausgesetzt und lange Reisen unternommen hätten:

Die Mitglieder der Akademie, welche Bereiche im Norden Europas und im Süden Amerikas bereisten, verfolgten mehr das Ziel, sie als Geometer denn als Philosophen zu besuchen. Da sie jedoch das eine wie das andere zugleich waren, darf man jene Regionen, die von den La Condamine und Maupertuis gesehen und beschrieben wurden, nicht als gänzlich unbekannt ansehen. ${ }^{3}$

Rousseau bezieht sich in dieser Passage auf jene beiden Expeditionen, die von der französischen Akademie ausgerüstet und ausgesandt wurden, um zu erkunden, ob die Erde rund wie eine Kugel - wie wir sie uns oft vorstellen und wie es der beleuchtete Globus in unserem Kinderzimmer suggerierte - oder am Äquator ausgebeult sowie an den Polen abgeplattet sei. Nun, bereits die Expedition von Maupertuis, des späteren Präsidenten der Preußischen Akademie der Wissenschaften, erzielte das Ergebnis, dass sich die Erdkugel entgegen der Annahmen unseres Kinderzimmers zu den Polen hin abplattet und daher vermutlich am Äquator ausgebuchtet ist. Die französischen Wissenschaftler, welche die zweite Expedition ins spanische Kolonialreich und ins heutige Ecuador führte, konnten

3 Rousseau, Jean-Jacques: Discours sur l'origine et les fondements de l'inégalité parmi les hommes. In (ders.): Euvres complètes. Bd. III. Edition publiée sous la direction de Bernard Gagnebin et Marcel Raymond avec, pour ce volume, la collaboration de François Bouchardy, Jean-Daniel Candaux, Robert Derathé, Jean Fabre, Jean Starobinski et Sven Stelling-Michaud. Paris: Gallimard 1975, S. 213. Soweit nicht anders angegeben, stammen alle Übersetzungen ins Deutsche vom Verfasser. Die originalsprachigen Zitate finden die Leserin und der Leser im Anhang. 
nachträglich diese Ausbuchtung belegen, welche zuvor bereits durch die Untersuchungen der Lappland-Expedition nahegelegt worden war. Kommen Sie heute nach Ecuador, so können Sie unweit von Quito das der französischen Expedition gewidmete Monument bestaunen, welches den Verlauf der Äquatoriallinie touristenwirksam nachzeichnet. Fein säuberlich trennt es die Nordhälfte unseres Planeten von seiner Südhälfte ab.

Doch nicht von den Geometern, sondern von den beteiligten französischen Philosophen wie Maupertuis oder La Condamine schien sich Rousseau bedeutsame Verbesserungen des Kenntnisstandes über die außereuropäische Welt zu erhoffen. Räumte der Bürger von Genf auch gerne Gehalt und Qualität mancher Reiseberichte ein, die im 18. Jahrhundert veröffentlicht worden waren, so verbarg er doch seine fundamentale Kritik am allgemeinen Niveau anthropologischer beziehungsweise ethnologischer Kenntnisse nicht. Dabei nahm er bei aller Bewunderung für das Werk Buffons auch den Bereich der Naturgeschichte und insbesondere der von ihren Vertretern genutzten Quellen nicht von dieser Kritik aus. Wir müssen heute einräumen, dass Rousseau mit seiner Kritik auf ganzer Linie Recht hatte.

Nach der Erwähnung einiger weniger glaubwürdiger Berichte stellte er mit aller wünschenswerten Deutlichkeit klar:

Sieht man von diesen Berichten einmal ab, so wissen wir nichts über die Völker Ostindiens, die von Europäern besucht wurden, die mehr das Füllen ihrer Börsen als ihrer Köpfe im Sinn hatten. [...] Die ganze Erde ist von Nationen bedeckt, von denen wir nur die Namen kennen, und wir machen uns anheischig, das ganze Menschengeschlecht zu beurteilen! Nehmen wir einmal an, ein Montesquieu, ein Buffon, ein Diderot, ein Duclos, ein d'Alembert, ein Condillac oder andere Männer diesen Zuschnitts würden Reisen unternehmen, um ihre Landsleute zu unterrichten, würden die Türkei, Ägypten, die Berberei, das Reich von Marokko, Guinea, die Kaffernländer, das Innere Afrikas und seine Ostküsten [...] und dann, in der anderen Hemisphäre, Mexico, Peru, Chile, die Magellanischen Gebiete, ohne die wahren oder falschen Patagonier zu vergessen, [...] beobachten und beschreiben, wie sie dies zu tun wissen (savent faire); nehmen wir weiter an, dass diese neuen Herkules, von ihren denkwürdigen Fahrten zurückgekehrt, nach ihrem Gutdünken danach die Natur-, Moral- und Politikgeschichte (Histoire naturelle, Morale et Politique) dessen, was sie gesehen hätten, verfassten, so würden wir selbst eine neue Welt aus ihren Federn entstehen sehen, und so würden wir lernen, die unsere zu kennen. ${ }^{4}$

Mit guten Gründen schätzte der Bürger von Genf die meisten europäischen Reisenden so ein, dass sie nicht den wissenschaftlichen Interessen ihrer Philosophen, sondern den kolonialen Interessen ihrer Mutterländer sowie der Ausbeutung der

4 Ebda., S. 213. 
eroberten beziehungsweise abhängigen Gebiete $\mathrm{zu}$ ihrem eigenen Wohl ergeben waren. Um die Mitte des 18. Jahrhunderts hatte eine zweite Phase beschleunigter Globalisierung begonnen, in welcher nun Frankreich und England die führenden europäischen Mächte waren, die Spanien und Portugal ablösten und eine neue Phase der Ausplünderung unseres Planeten zwecks Bereicherung der jeweiligen europäischen Mutterländer begann. Ich sprach von dieser Phase, als ich den Auftakt des 19. Jahrhunderts von einer Periode beschleunigter Globalisierung gerahmt nannte.

So entgehen die meisten Reisenden aufgrund ihrer persönlichen und kommerziellen Interessen dem vernichtenden Urteil des Genfer Philosophen nicht. Rousseau verfügte über ein genaues Gespür für die politischen, sozialen und auch wirtschaftlichen Entwicklungen seiner Zeit. Doch der Verfasser des Diskurses über die Ungleichheit leugnete keineswegs die fundamentale Bedeutung der Reisen für die Ausweitung menschlicher Kenntnisse, ganz im Gegenteil! Selbst in seinen Roman Julie ou la Nouvelle Héloïse baute er eine Passage ein, in welcher er seinen Helden Saint-Preux auf eine Weltumsegelung schickte, die selbstverständlich an Bord eines englischen Schiffs stattfand. Ein hellwaches Bewusstsein für die Veränderungen seiner Zeit zeichnete ihn als Schriftsteller wie als „Weltweisen“ - wie man Philosophen früher auf Deutsch nannte - aus.

So ging es ihm darum, in möglichst verschiedene Himmelsrichtungen unterschiedliche „philosophes“ (im Sinne des 18. Jahrhunderts) auszusenden, die nicht nur über ein Wissen („savoir“), sondern mehr noch über ein „savoir faire“ und ein „savoir voir“ verfügten und in der Lage wären, nach ihrer Rückkehr nach Europa das, was sie gesehen hatten, an ihre Landsleute weiterzugeben. „Savoir faire“ und „savoir voir“ würden auf diese Weise für die künftigen Leser des Reisenden in ein „savoir faire voir“, ein Wissen und eine Technik des Vor-Augen-Führens, umschlagen, das nicht nur die europäische Sichtweise der Neuen Welt modifizieren und den „Nouveau-Monde“ in einen „monde nouveau“ verwandeln, sondern den Blick auch auf die europäischen Länder selbst grundlegend verändern könnte. Man kann Rousseau für eine derartige Sichtweise, die von der europäischen Aufklärung zu keinem Zeitpunkt eingelöst oder verwirklicht wurde, nur bewundern.

In der angeführten Passage erscheinen Sehen und Schreiben als komplementäre Handlungen, die in ihrer Abfolge einen Sinn (bezüglich) der Neuen Welt hervorbringen, den Rousseau zu glauben bereit wäre: „il faudra les en croire“. ${ }^{5}$ Doch gründet dieser Glaube für Rousseau nicht auf dem Schreiben, der „écriture“. Nicht die Bewegung des Diskurses, sondern die Bewegung des Reisens, das eine direkte Sicht auf die Dinge gewährt, jene Ortsveränderung also, die ein unmittel-

5 Ebda., S. 214. 
bares Sehen des Anderen ermöglicht, verleiht dem Schreiben über das Andere Autorität und damit erst eigentliche Autorschaft im vollen Sinne. Es ist faszinierend zu sehen, wie „Jean-Jacques“, einer der großen Stammväter der Romantik, bereits jene Herausforderungen zu erspüren und benennen versuchte, welche das sich an ihn anschließende Jahrhundert wesentlich mitprägen sollten.

In der zitierten Anmerkung zu seinem zweiten Discours hat Rousseau, dem wir im Verlauf der Vorlesung noch an mehreren Stellen und Kreuzungspunkten begegnen werden, darauf aufmerksam gemacht, dass der Universalismus der Aufklärung letztlich auf einem nur dürftigen Faktenwissen aufruht. Zudem wies er darauf hin, dass insbesondere die Kenntnis des Menschen und damit die Anthropologie Bereiche darstellen, auf denen die Wissenschaften oder besser noch die Wissensbestände Europas und der europäischen Philosophen erhebliche Lücken aufwiesen. Um also eine Geschichte des Ursprungs der Ungleichheit unter den Menschen entwerfen zu können - und damit eine Menschheitsgeschichte schlechthin -, wie es Rousseau unternahm, war eine Verknüpfung von Erfahrungswissen mit Theorie, konkreter Kenntnis verschiedenster Kontexte mit auf Fakten basierender Erkenntnis, der Vielheit der Erscheinungen mit der Einheit des Denkens notwendig. Erst dadurch können für die Menschheitsgeschichte generell allgemeinere und fundiertere Aussagen getroffen werden. Freilich ist es durchaus zutreffend, dass der Entwurf zahlreicher Universalismen der Aufklärung gerade auf dem Fehlen eines Faktenwissens wie eines Lebens- und Erlebenswissens beruhte, deren Existenz in vielen Fällen die Abfassungen allgemeiner Theorien nur behindert und gestört hätte.

In diesen Sätzen Rousseaus drückt sich zudem aus, dass der Universalismus der Aufklärung nun einer konkreteren Auseinandersetzung, eines faktenbezogeneren Wissens bedurfte, um nicht bloß auf abstrakte Prinzipien und Gesetzmäßigkeiten reduziert $\mathrm{zu}$ bleiben. Mit anderen Worten: Es ging nunmehr darum, dem abstrakten Weltbürger, dem „citoyen du monde“, auch eine tatsächliche Weltkenntnis zu vermitteln und aus dieser Perspektive die verschiedenen Teile und Regionen unseres Planeten zusammenzudenken. Aus heutiger Sicht ließe sich dem vielleicht hinzufügen, dass ein stärker faktenbezogenes Wissen eine abstrakte Einheit des Denkens unterlaufen konnte, so dass sich hieraus ein Denken der Differenz - oder doch zumindest ein erstes Denken von Differenz - zu entwickeln vermochte. Rousseau formulierte in seiner wichtigen Anmerkung Überlegungen, die freilich erst das 19. Jahrhundert einlösen sollte, indem es Figuren wie Alexander von Humboldt hervorbrachte, die - um in Rousseaus Diktion zu bleiben - als Philosophen und Geometer, folglich als Natur- und Kulturforscher unsere Erde weit außerhalb der Grenzen Europas bereisen sollten. Doch dazu später mehr!

Ich möchte Ihnen gerne an dieser Stelle einige weitere Überlegungen aus dem Bereich der Philosophie vortragen, mit Hilfe derer Sie vielleicht leichter erkennen 
können, dass das Spannungsfeld von Lokalem und Regionalem einerseits, von Internationalem und Globalem andererseits eine durchgängige Unterströmung des ausgehenden 18. Jahrhunderts wie der ersten Hälfte des 19. Jahrhunderts und damit auch der Romantik in beiden Welten war. Dabei greife ich auf einen Philosophen zurück, der ganz im Gegensatz zu dem von Rousseau Geforderten seine eigene Heimat nie verließ, sein geliebtes Königsberg im damaligen Ostpreußen, wo er selbst die Routen seiner täglichen Spaziergänge niemals veränderte, da es ihm sonst schwindelig werden konnte. Doch diesem Manne nun wurde erstaunlicherweise niemals schwindelig, wenn er auf Grundlage seiner Bibliothek, seines immensen, aus Büchern zusammengetragenen Wissens eine Welt konstruierte, welche die seine und doch zugleich auch die unsrige war und ist. Gäbe es ein besseres und ansprechenderes Beispiel als diesen ostpreußischen Philosophen, um erkennbar aufzuzeigen, dass es sehr wohl möglich war, die ganze Welt universalistisch aus einer - weltgeschichtlich betrachtet - eher marginalen Ecke unseres Planeten zu konstruieren?

Gerade die Frage des Zusammenspiels von Universalem und Partikularem oder, wie wir aus heutiger Sicht ebenfalls sagen könnten, aus Lokalem und Globalem beschäftigte die führenden Köpfe am Ausgang des 18. und zu Beginn des 19. Jahrhunderts unter dem Eindruck der fortgesetzten zweiten Phase beschleunigter Globalisierung ungeheuer. Diese einen unbeschwerten Universalismus gründlich unterlaufende Tatsache ist aus der Sicht nationalliterarischer und nationaler Geschichtsschreibung häufig nachträglich ausgeblendet worden; und gerade sie gilt es zu rekonstruieren, um ein neues Bild der Romantik herauszuarbeiten.

Denn bereits 1784 - also zwei Jahre vor dem Tod Friedrichs II. von Preußen, dessen „verlassene Paläste“ Alexander von Humboldt 1792 besuchte - hatte Immanuel Kant in seiner Idee zu einer allgemeinen Geschichte in weltbürgerlicher Absicht den Versuch unternommen, derartige Fragestellungen durch den Entwurf einer politischen Neuordnung partikularer und stets ungleicher Machtsysteme zu erweitern. Auch ohne allzu viele Kriege, Verwüstungen und Notlagen, so der große Philosoph aus Königsberg, hätte uns die Vernunft schon lange einen gangbaren Weg weisen können. Davon ausgehend entfaltete er seinen großen Entwurf dessen, was später einmal die - freilich unvollkommene - Institution der Vereinten Nationen sein sollte:

[So hätte uns die Vernunft schon lange sagen können:] aus dem gesetzlosen Zustande der Wilden hinaus zu gehen, und in einen Völkerbund zu treten; wo jeder, auch der kleinste, Staat seine Sicherheit und Rechte, nicht von eigener Macht, oder eigener rechtlichen Beurteilung, sondern allein von diesem großen Völkerbunde (Foedus Amphictyonum), von einer vereinigten Macht, und von der Entscheidung nach Gesetzen des vereinigten Willens, erwarten könnte. So schwärmerisch diese Idee auch zu sein scheint, und als eine solche 
an einem Abbé von St. Pierre oder Rousseau verlacht worden (vielleicht, weil sie solche in der Ausführung zu nahe glaubten): so ist es doch der unvermeidliche Ausgang der Not [...]. Obgleich dieser [künftige große] Staatskörper für itzt nur noch sehr im rohen Entwurfe dasteht, so fängt sich dennoch gleichsam schon ein Gefühl in allen Gliedern, deren jedem an der Erhaltung des Ganzen gelegen ist, an zu regen; und dieses gibt Hoffnung, dass, nach manchen Revolutionen der Umbildung, endlich das, was die Natur zur höchsten Absicht hat, ein allgemeiner weltbürgerlicher Zustand, als der Schoß, worin alle ursprüngliche Anlagen der Menschengattung entwickelt werden, dereinst einmal zu Stande kommen werde. ${ }^{6}$

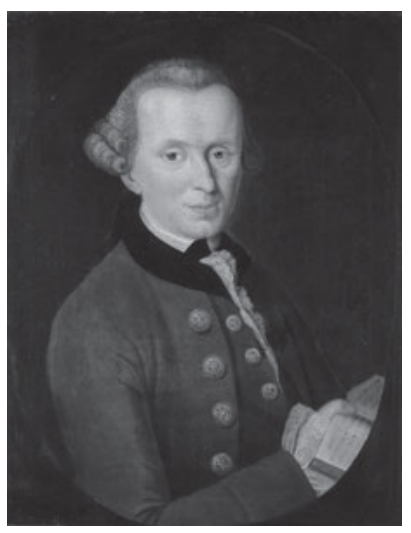

Abb. 2: Immanuel Kant

(Königsberg, Preußen, 1724 - ebenda, 1804).

Es ist aufschlussreich, dass Immanuel Kant, der Verfasser der Kritik der reinen Vernunft, an dieser Stelle offenkundig einen Weg wählt, auf dem er sich nicht als erster wähnt und von dem er zugleich ebenfalls weiß, dass er sich der Gefahr aussetzen könnte, wie die Herren Rousseau und Bernardin de Saint-Pierre (von denen er im Übrigen wenig hielt) verspottet zu werden. Hierbei ist bemerkenswert, dass sich Kant damit auf zwei der großen Gestalten nicht nur der französischen Romantik bezieht, um seine universale Ausrichtung und das von ihm entworfene Bild eines großen weltumspannenden Staatskörpers weiterzudenken. In diesen Überlegungen des Königsberger Philosophen zeigt sich erneut, dass eine durchgängige Unterströmung die Denker der Aufklärung und der Spätaufklärung vereint und dass sich ein solches Denken im weiteren Verlauf dann auch in die zum damaligen Zeitpunkt in der Sattelzeit befindliche Romantik hinein fortpflanzen sollte: Der Universalismus der Kant'schen Überlegungen spricht für sich.

6 Kant, Immanuel: Idee zu einer allgemeinen Geschichte in weltbürgerlicher Absicht. In: Weischedel, Wilhelm (Hg): Immanuel Kant: Werkausgabe in 12 Bänden. Bd. 11: Schriften zur Anthropologie, Geschichtsphilosophie, Politik und Pädagogik 1, Frankfurt a.M.: Suhrkamp 1977, S. 42 u. 47. 
Diese Idee eines Völkerbundes, deren konkrete politische Umsetzungsversuche uns in ihren Stärken und Schwächen ein Gutteil unseres 20. und des beginnenden 21. Jahrhunderts begleitet haben und auch heute noch in Atem halten, wird von Kant in jenem künftigen Status verankert, den er als ,allgemeiner weltbürgerlicher Zustand“ bezeichnete. Wie sehr diese Überlegungen zu einer künftigen Weltpolitik, die diesen Namen auch verdiente, noch in heilsgeschichtlichen Vorstellungen wurzeln, zeigen Formulierungen von der „tröstende[n] Aussicht in die Zukunft“, „in welcher die Menschengattung in weiter Ferne vorgestellt wird, wie sie sich endlich doch zu dem Zustande emporarbeitet, in welchem alle Keime, die die Natur in sie legte, völlig können entwickelt und ihre Bestimmung hier auf Erden kann erfüllet werden“. ${ }^{7}$ Es ist keine Frage, dass die Grundlagen einer künftigen weltumspannenden Ordnung und Welt-Innenpolitik die Formen und Normen abendländischen Denkens sein sollten. Dies bildet die unhinterfragte und unhinterfragbare Voraussetzung für die Weltentwürfe nicht allein Immanuel Kants, sondern der europäischen Aufklärung überhaupt.

Dass der Philosoph aus Königsberg in seinen universalistischen Entwürfen bisweilen unbestreitbar rassistische Ausfälle einflocht und insbesondere die Schwarzen des afrikanischen Kontinents unbeschreiblich herabwürdigte, ist im Verlauf des 19. Jahrhunderts und auch über lange Phasen des 20. Jahrhunderts weitgehend - soweit ich sehe - unkommentiert geblieben. Der Rassismus eines Immanuel Kant konnte ein abendländisches Denken nicht stören (sondern bestenfalls bestätigen), das sich im Verlauf des 19. Jahrhunderts mehr denn je seiner technisch-materiellen und militärischen Überlegenheit bewusst wurde und zunehmend alle Areas dieser Erde als potenzielle Kolonialgebiete ansah. Die weltumspannende Überlegenheit der Europäer, aber auch die unerhörte Ausbeutbarkeit der Erde durch den europäischen Kolonialismus schienen keine Grenzen mehr zu kennen und fanden in den Rassismen dieses Kopfes der deutschen Aufklärung wie in den Schriften vieler anderer Philosophen der „Lumières“ ihre wohlfeile Bestätigung.

Erst in neuerer Zeit ist der Rassismus des Königsberger Philosophen zunehmend in den Fokus gerückt. Dabei wurde auch die Tatsache deutlich, dass Kant nicht allein abstrakt die intellektuellen Bildungsfähigkeiten von Schwarzen in der afrikanischen Welt in Frage stellte, sondern auch die großen Erfolge des ersten schwarzen Philosophen an einer preußischen Universität mit Schweigen überging. Die blitzgescheiten Entwürfe eines Anton Wilhelm Amo in Halle, Wittenberg oder Jena wollten nicht in sein rassistisches Konzept der Philosophie

7 Ebda., S. 49. 
passen. ${ }^{8}$ So besitzt der vermeintlich leuchtende Universalismus eines Immanuel Kant angesichts seiner dürren Datengrundlage und der Geste des Totschweigens eines ihm unliebsamen Philosophen afrikanischer Herkunft einen mehr als bitteren Beigeschmack.

Doch nochmals zurück zu Kants Idee zu einer allgemeinen Geschichte in weltbürgerlicher Absicht aus dem Jahre 1784! Denn es mögen derlei Formulierungen und die Betonung einer erst in weiter Ferne liegenden Erfüllung des weltbürgerlichen Zustands gewesen sein, welche die Zeichentheoretikerin und Psychoanalytikerin Julia Kristeva zur ihrer eigenen Kant-Lesart veranlassten. In ihrer bisweilen etwas verkürzenden, Kant allzu sehr auf die französischen Denktraditionen reduzierenden Analyse bezog sie einerseits den Verfasser der Kritik der reinen Vernunft auf die kosmopolitischen Entwürfe der Encyclopédie sowie den Enthusiasmus der politischen Vordenker der Französischen Revolution, ${ }^{9}$ und hob andererseits den kühnen und utopischen Charakter des Kosmopolitismus-Gedankens der „Lumières“ heraus. ${ }^{10}$ Für Immanuel Kant freilich war die allgemeine Geschichte in weltbürgerlicher Absicht keineswegs von Analysen und Befunden empirischer Forschung an konkreten (und keineswegs u-topischen) Orten getrennt. Seinen Vorschlägen fügte er durchaus relativierend hinzu:

Dass ich mit dieser Idee einer Weltgeschichte, die gewissermaßen einen Leitfaden a priori hat, die Bearbeitung der eigentlichen bloß empirisch abgefaßten Historie verdrängen wollte: wäre Mißdeutung meiner Absicht; es ist nur ein Gedanke von dem, was ein philosophischer Kopf (der übrigens sehr geschichtskundig sein müßte) noch aus einem anderen Standpunkte versuchen könnte. ${ }^{11}$

In Alexander von Humboldts Werken können wir sehr wohl den Versuch erkennen, in der Tat von „einem anderen Standpunkte“ aus eine derartige Weltgeschichte in Angriff zu nehmen und den Planeten - freilich von einer fundierten Datenbasis aus - als ineinander greifendes System Erde weltweit zu denken. Dieser philoso-

8 Vgl. hierzu Ette, Ottmar: Anton Wilhelm Amo: Philosophieren ohne festen Wohnsitz. Eine Philosophie der Aufklärung zwischen Europa und Afrika. Mit einem Vorwort zur zweiten Auflage. Berlin: Kulturverlag Kadmos 2020.

9 Kristeva, Julia: Etrangers à nous-mêmes. Paris: Gallimard 1988, S. 251: „Et Kant, comme les cosmopolites fougueux de la Révolution, mais avec la précision logique d'une argumentation apaisée [...].“

10 Ebda., S. 213: „Avec son envers enragé et son endroit généreux, de Fougeret à Montesquieu, le cosmopolitisme apparaît désormais comme une audace, utopique pour le moment, mais avec laquelle doit compter une humanité consciente de ses limites et aspirant à les dépasser dans l'organisation des liens sociaux et des institutions.“

11 Kant, Immanuel: Idee zu einer allgemeinen Geschichte in weltbürgerlicher Absicht, S. 49s. 
phische Kopf, der stets seine Forschungen empirisch zu fundieren suchte, hatte im Herbst 1791 noch aus Freiberg an Paul Usteri geschrieben, er arbeite bereits seit zwei Jahren an einer „Geschichte der Pflanzenwanderungen“, die er keineswegs als eine rein pflanzengeographische oder botanische Arbeit, sondern als „Ausarbeitung dieses so vernachlässigten Theils der Universalgeschichte“ verstand. ${ }^{12}$ Der jüngere der beiden Humboldt-Brüder sah sich durchaus in den Fußstapfen der Aufklärung, verwarf jedoch bei aller Anlehnung schon früh deren weitgehend faktenfreie Grundlagen und ging hart mit einer sich insbesondere in französischer Sprache ausdrückenden Philosophie zu Gericht, welcher er blindes „Systemdenken" vorwarf.

Denn bereits beim zwanzigjährigen Humboldt entwickelte sich ein Forschungsprogramm, das sich an der Erhellung der Weltgeschichte und ihrer globalen Zusammenhänge orientiert und von einer - im Sinne Kants - „weltbürgerlichen Absicht" geleitet ist. Diese kosmopolitische Dimension seines Forschens und Denkens wird sich, wenn auch anders als bei dem ihn früh schon beeinflussenden Kant angelegt, als Leitfaden durch die Schriften dieses „citoyen de l'univers“ bis hin zu seinem Kosmos ziehen. In einem Brief vom 3. Oktober 1790 sprach er gegenüber Johann Reinhold Forster, dem Vater Georg Forsters, unverkennbar mit Blick auf den Königsberger Philosophen von dem „Lichte, was jetzt so unaufhaltsam (ich möchte sagen, aus seinem ehemaligen latenten Zustande) vom Norden her einbricht". ${ }^{13}$

Alexander von Humboldt verstand sich als Weltbürger, und er war es durchaus in der Tradition des 18. Jahrhunderts, freilich mit jener empirischen und reisetechnischen Basis, die Rousseau von den künftigen Philosophen abverlangt hatte; Vorstellungen und Forderungen, die sicherlich einem Kant eher fremd gewesen wären. Der Erforscher von Natur und Kultur ${ }^{14}$ bewahrte sich diesen von der Aufklärung ererbten, aber von ihm mit Hilfe seiner Kosmopolitik weiterentwickelten Hang zum Weltbürgerlichen selbst noch gegen Ende seines Lebens in der immer enger werdenden Atmosphäre des preußischen Königshofes in Berlin und Potsdam. Er tat dies inmitten eines Denkens, in welchem sich die Grundlagen der künftigen Arroganz wie der künftigen Katastrophen Preußens und Deutschlands bereits abzeichneten.

12 Humboldt, Alexander von: Die Jugendbriefe. 1787-1797. Herausgegeben und erläutert von Ilse Jahn und Fritz G. Lange. Mit einem Vorwort von Kurt-R. Biermann. Berlin: Akademie Verlag 1973, S. 163 u. 164.

13 Ebda., S. 109.

14 Vgl. hierzu Ette, Ottmar: Natur und Kultur: Lebenswissenschaftliche Perspektiven Humboldtscher Wissenschaft. In: Ette, Ottmar / Drews, Julian (Hg.): Horizonte der Humboldt-Forschung. Natur, Kultur, Schreiben. Hildesheim - Zürich - New York: Georg Olms Verlag 2016, S. 13-51. 
Was aber ist im Sinne der europäischen Aufklärung des 18. Jahrhunderts ein Weltbürger? Voltaires Dictionnaire philosophique enthält zwar keinen direkten Eintrag unter Stichwörtern wie „cosmopolite“ oder „cosmopolitisme“, präsentiert aber eine für uns wertvolle Äußerung am Ende des Eintrags zu „Patrie“, die hier angeführt sei:

Denn so ist die conditio humana beschaffen, dass die Größe des eigenen Landes zu wünschen heißt, den Nachbarn Übles zuzudenken. Wer wünschte, dass sein Vaterland niemals größer noch kleiner, niemals reicher noch ärmer wäre: Der wäre der Bürger des Universums. ${ }^{15}$

Sicherlich darf man gemäß der Denkräume des französischen 18. Jahrhunderts durchaus behaupten, dass dieses ,Universum“ wohl nur wenig die Dimensionen Europas überschritten haben dürfte. Doch war in der „République des Lettres“, für die Voltaire schrieb und für die auch sein Dictionnaire philosophique gedacht war, ganz selbstverständlich ein Binnengrenzen überschreitendes Europa gemeint, das man mit dem Aufklärungsforscher Pierre Chaunu als das „Europe des Lumières “ bezeichnen könnte. ${ }^{16}$

Das in gewisser Weise realisierte Ideal des von Voltaire so apostrophierten „citoyen de l'univers“ findet sich in einer Passage des 26. Kapitels von Humboldts Relation historique in aller wünschenswerten Deutlichkeit. Im Kontext seiner Erörterungen über die künftigen Beziehungen zwischen Europa und Amerika worunter er im Gegensatz bereits zu manchen seiner Zeitgenossen und dem heute vorherrschenden Sprachgebrauch nicht die Vereinigten Staaten verstand - und seiner Prognosen bezüglich der künftigen Entwicklung der erfolgreich um ihre Unabhängigkeit kämpfenden spanischen Kolonien stellte er fest:

Gewiss ist infolge der großen Umwälzungen, denen die menschlichen Gesellschaften unterliegen, das Gesamtvermögen, und damit das gemeinschaftliche Erbgut der Zivilisation, unter die Völker beider Welten ungleich verteilt; aber allgemach stellt sich das Gleichgewicht wieder her, und es ist ein verderbliches, ja ich möchte sagen gottloses Vorurteil zu meinen, es sei ein Unheil für das alte Europa, wenn auf irgendeinem anderen Teil unseres Planeten der öffentliche Wohlstand gedeiht. Die Unabhängigkeit der Kolonien wird nicht zu ihrer Isolierung führen, sie werden vielmehr dadurch den Völkern von alter Kultur nähergebracht werden. Der Handel wirkt naturgemäß dahin, zu verbinden, was eine eifersüchtige Politik so lange auseinandergehalten. Noch mehr: Es liegt im Wesen der Zivilisation, dass sie sich ausbreiten kann, ohne deshalb da, von wo sie ausgegangen, zu erlöschen. Ihr allmähliches

15 Voltaire: Dictionnaire philosophique. Bd. IV (Euvres complètes de Voltaire, Bd. XX). Paris: Garnier Frères 1879, S. 185 f.

16 Vgl. die klassische Studie von Chaunu, Pierre: La Civilisation de l'Europe des Lumières. Paris: Flammarion 1982. 
Vorrücken von Ost nach West, von Asien nach Europa, beweist nichts gegen diesen Satz. Ein starkes Licht behält seinen Glanz, auch wenn es einen größeren Raum beleuchtet. Geistige Kultur, die fruchtbare Quelle des nationalen Wohlstands, teilt sich durch Berührung mit; sie breitet sich aus, ohne von der Stelle zu rücken. ${ }^{17}$

Diese von Alexander von Humboldt in der zweiten Hälfte der 1820er Jahre niedergeschriebenen Sätze verweisen uns auf eine Kontinuität ethisch fundierten Weltbewusstseins, ${ }^{18}$ das weit in die nicht nur deutsche Romantik hineinragt und in einen eigenartigen Kontrast zu der sonst für die Romantik in Anschlag gebrachten nationalen bis nationalistischen Tendenz tritt. Humboldts Denken steht gewiss in einer Beziehung zu einem wirtschaftsliberalistischen Denken im Sinne von Adam Smith, das zweifellos im 18. Jahrhundert und in der schottischen Aufklärung seinen Wurzelgrund besitzt. Es sollte die nationalökonomischen und wirtschaftsliberalen Vorstellungen vor allem des 19. Jahrhunderts prägen. Die Idee einer einzigen Menschheit, wie sie die europäische Aufklärung - aber auch die Aufklärung in den amerikanischen Kolonien Spaniens - entwickelt hatte, ging nicht mit dem Ende der „Ilustración“, nicht mit dem Ende des 18. Jahrhunderts unter. Und sie stellte vor allem jene Frage nach dem Weltethos, die sich in Kants Überlegungen zum Völkerbund, zu einem großen Staatskörper, findet und ihren konkreten Ausfluss im Völkerbund der 20er und 30er Jahre und nach Ende des Zweiten Weltkrieges schließlich im Aufbau der Vereinten Nationen fand.

In diesem Sinne war Alexander von Humboldt zweifellos ein Erbe der europäischen wie der amerikanischen Aufklärung - und immer, wenn ich „Amerika“ oder ,amerikanisch“ sage, sind damit nicht die USA gemeint, sondern der gesamte amerikanische Kontinent, den die Vereinigten Staaten inhaltlich im westlichen Gebrauch dieses Begriffes usurpiert haben. Humboldt ging von einem grundlegenden Gleichgewicht zwischen den Völkern aus, als deren Bindeglied der Handel und wechselseitiger Austausch fungieren sollten. Er war zutiefst von der gegenseitigen Befruchtung der Kulturen wie der Nationen überzeugt und vertrat überdies antikolonialistische Vorstellungen, die ihn nicht an der Befreiung und Unabhängigkeit zu seiner Zeit noch abhängiger, kolonisierter Regionen unseres Planeten zweifeln ließen.

17 Humboldt, Alexander von: Reise in die Äquinoktial-Gegenden des Neuen Kontinents. Herausgegeben von Ottmar Ette. Mit Anmerkungen zum Text, einem Nachwort und zahlreichen zeitgenössischen Abbildungen sowie einem farbigen Bildteil. 2 Bde. Frankfurt am Main - Leipzig: Insel Verlag 1991, hier Bd. 2, S. $1465 \mathrm{f}$.

18 Vgl. Ette, Ottmar: Weltbewusstsein. Alexander von Humboldt und das unvollendete Projekt einer anderen Moderne. Mit einem Vorwort zur zweiten Auflage. Weilerswist: Velbrück Wissenschaft 2020. 
Für unsere Vorlesung sind in diesen Worten Alexander von Humboldts viele Aspekte spannend: nicht nur seine Rede von den „beiden Welten“, mit denen er die Wichtigkeit der transatlantischen Beziehungen zwischen den Amerikas und Europa hervorhob, sondern auch seine feste Überzeugung, dass es dereinst einen fundamentalen Interessen-, Handels- und Machtausgleich geben werde. Die Humboldt'sche Kosmopolitik zielte auf weltweite Balance und ein gemeinsames Fortschreiten der Menschheit ab, wobei er sich eine Welt der Zukunft vorstellte, in welcher es schon bald keine Sklaverei, keinen Kolonialismus und keine Abhängigkeiten mehr geben sollte. Kurz: Eine gerechte Weltordnung sollte und würde heraufziehen, von der alle Nationen und Völker gleichmäßig profitieren. Die pure Utopie, meinen Sie?

Vielleicht wären wir einer solchen Epoche in der Menschheitsgeschichte zu einem Zeitpunkt sehr nahe gekommen, als der Kalte Krieg und die Konfrontation der Blöcke Ende der achtziger Jahre des 20. Jahrhunderts urplötzlich ein Ende fand: Da gab es die einmalige Chance. Doch die USA haben dies als „Sieg im Kalten Krieg“ gedeutet und eine Ausplünderung unseres Planeten forciert, welche die Probleme, inmitten derer wir uns heute befinden, nicht geschaffen haben, wohl aber beschleunigten. Derartige Epochen - Zeitfenster, in denen sich ein grundlegender Wandel vollziehen könnte - sind selten in der Menschheitsgeschichte. Wer sie nicht nutzt, sondern - lange vor einem Donald Trump, der den Niedergang der USA als oligarchischer Statthalter nur noch beschleunigt - auf ein „America first“ setzt, muss sich später nicht wundern, wenn die Weltgeschichte an ihm vorbei geht und mit Recht andere Mächte die Weltbühne betreten.

Mir scheint es vor dem Hintergrund unserer aktuellen Situation keineswegs müßig und unnütz zu sein, diese Vorstellungen an den Beginn unserer Vorlesung zu stellen. Denn wir müssen diese weithin ausgeblendete Vorgeschichte der Romantik in Erinnerung behalten, um besser zu verstehen, dass die Romantik ein überaus komplexes Beziehungsgeflecht ist, so dass wir sie nicht auf eine ganz bestimmte, von den nationalliterarischen Geschichtsschreibern bewusst intendierte nationale Zwergen-Gestalt beschränken sollten. Sie lässt sich eben nicht so wohlfeil reduzieren auf einen Gegenschlag des Pendels gegen den Universalismus der Aufklärung, auf eine Rückwärtsbewegung ins Partikulare, die den Nährboden für die unterschiedlichen europäischen und nicht-europäischen Nationalismen dargestellt hätte. Wir müssen uns von der Dominanz derartiger Vorstellungen freimachen, um deren reale Bedeutung adäquat einschätzen zu können.

Zugleich erlauben uns diese Überlegungen, unsere heutige Situation nach der Wende zu einem neuen Jahrtausend und nach dem Ende der vierten Phase beschleunigter Globalisierung besser zu verstehen als eine keineswegs - wie man bisweilen lesen kann - postglobale Zeit, sondern vielmehr als Epoche, welche sich zwischen zwei Phasen beschleunigter Globalisierung ansiedelt. Denn weite 
Strecken des 19. Jahrhunderts waren genau dies: viele Jahre und lange Jahrzehnte, welche sich genau zwischen zwei Phasen beschleunigter Globalisierung situieren.

So möchte ich an dieser Stelle einen kurzen Bezug zu einem der um die Jahrtausendwende weltweit führenden Anthropologen herstellen. Clifford Geertz versuchte in seinen 1996 gehaltenen Wiener Vorlesungen, die Situation nach 1989, nach dem Fall der Berliner Mauer und nach Ende des Kalten Krieges zu analysieren und deren Konsequenzen für unser heutiges Denken jenseits bipolarer Blockbildungen auszuloten. Diese Überlegungen, die vor einem Vierteljahrhundert angestellt wurden, sind - wie ich finde - für uns noch immer von großem Interesse. Ich will Ihnen gerne eine Passage aus der ersten Vorlesung präsentieren, welche die Situation des Jahres 1996, aber vielleicht auch noch des heutigen Tages in ihrer Zukunftsoffenheit recht gut auf den Punkt bringt:

Heute, sieben Jahre nach dem Fall der Berliner Mauer, scheinen wir uns einmal mehr an einem solchen Ort und in einer solchen Zeit zu befinden. Die Welt, in der wir seit Teheran und Potsdam, oder eigentlich seit Sedan und Port Arthur, gelebt haben - eine Welt der kompakten Mächte und der rivalisierenden Blöcke, der Formierung und Neuformierung von Makroallianzen - gibt es nicht mehr. Doch was an ihre Stelle getreten ist und wie wir uns damit auseinanderzusetzen haben, ist entschieden weniger klar.

Ein weit pluralistischeres Muster der Beziehungen zwischen den Völkern der Welt scheint im Entstehen, aber seine Form bleibt vage und unregelmäßig, stückhaft und bedrohlich unbestimmt. [...] Diese und andere, aus ihnen hervorgegangene Entwicklungen (ethnische Bürgerkriege, sprachliche Separatismen, „Multikulturalisierung“ des internationalen Kapitals) haben kein Bild einer neuen Weltordnung entstehen lassen. Sie haben im Gegenteil den Eindruck der Zerstreuung und der Partikularität, der Komplexität und Dezentriertheit bestärkt. Die Symmetrien des Schreckens, wie sie die Nachkriegsära prägten, haben sich aufgelöst, und uns, so scheint es, bleiben nur deren Bruchstücke. ${ }^{19}$

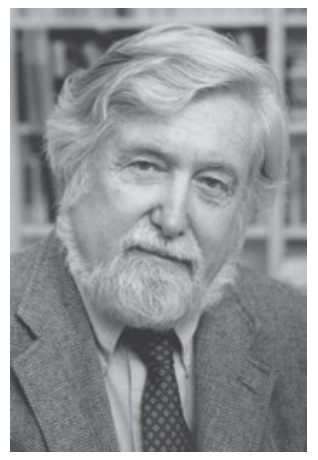

Abb. 3: Clifford James Geertz

(San Francisco, 1926 - Philadelphia, 2006).

19 Geertz, Clifford: Welt in Stücken. Kultur und Politik am Ende des 20. Jahrhunderts. Wien: Passagen 1996, S. $16 \mathrm{f}$. 
Die sorgfältige und zugleich vorsichtige Analyse von Clifford Geertz ist in vielfacher Hinsicht interessant - nicht nur für unsere aktuelle Situation und Lage, sondern auch für unseren aktuellen Blick auf die Geschichte. Wenn es richtig ist, dass wir es heute mit einer „Welt in Stücken“ zu tun haben, die nicht mehr jener alten antagonistisch-bipolaren Logik folgt oder zumindest dominant gehorcht, nach der sich letztlich alles am großen Ost-West-Gegensatz ausrichtete, wird klar, dass jener Traum der europäischen Moderne von einer raschen Homogenisierung oder zunehmenden Entdifferenzierung der Welt bestenfalls eine Seite der Medaille ist. Die andere Seite ist just in der paradoxerweise umgekehrt verlaufenden Entwicklungsrichtung eingeschrieben. Was ist damit gemeint?

Wir sehen unzweideutig einen Prozess zunehmender Differenzierung in kultureller, religiöser, ideologischer und sozialer Hinsicht und zugleich eine Entwicklung hin zu immer kleineren staatlichen Einheiten, der gerade für die Deutschen vielleicht stärker als für andere Völker von einer doppelten Bewegung der Vereinheitlichung verdeckt wurde. Zum einen war dies die sogenannte ,Wiedervereinigung' des Jahres 1989 - es hatte nie zuvor einen solchen deutschen Staat mit diesen Grenzen gegeben -, welche zugleich auch eine neue Phase der Globalisierung beschleunigte. Zum anderen gab es den europäischen Einigungsprozess, dessen Dynamik jedoch nicht verbergen konnte, dass wir am Ende des 20. Jahrhunderts fast viermal mehr Nationen haben als um die Mitte desselben Jahrhunderts, und dass innerhalb dieses wunderbaren Einigungsvorgangs Europas neue Bruchlinien entstanden, von denen der Brexit des Vereinigten Königreichs nur die offensichtlichste war. Die Zahl unabhängiger oder nach Unabhängigkeit strebender Nationalstaaten steigt - auch in Europa - weiter an und lässt die augenblickliche Epoche in ein eigenartiges Verhältnis zu jenen geschichtlichen Entwicklungen treten, welche gerade den Beginn des 19. Jahrhunderts prägten. Im Bereich der Literatur lassen sich diese Entwicklungen weitaus lebensnaher, als gelebte Entwicklungen, nachvollziehen als in den eher trockenen Geschichtsstunden. Denn die Literaturen bieten uns die Möglichkeit, all das geradezu hautnah zu erleben und nachzuerleben, was die Historie an allgemeinen Vorgängen und abstrakten Prozessen hervorgebracht hat.

Werfen wir aus dieser zugegebenermaßen etwas ungewöhnlichen Perspektive einen Blick auf die Romantik, so zeigt sich, dass der so eklatante Gegensatz zwischen Aufklärung und Romantik als unverkennbare Opposition, aber auch als ein mit einer gewissen dialektischen Dynamik ausgestattetes Kontinuum gedacht werden kann. Denn wenn wir mit Jürgen Habermas das Projekt der Moderne mit dem letzten Drittel des 18. Jahrhunderts beginnen lassen, ${ }^{20}$ dann

20 Vgl. Habermas, Jürgen: Die Moderne - ein unvollendetes Projekt (1980). In (ders.): Kleine Politische Schriften (I - IV). Frankfurt am Main: Suhrkamp 1981, S. 444-466. 
ist die Romantik sicherlich kein Teil einer Gegenbewegung gegen diese Moderne, sondern ein in eine dialektische Bewegung eingebundener Prozess einer sich verstärkenden, vielleicht sogar radikalisierenden Moderne. Dabei handelte es sich um einen historischen und kulturellen Entwicklungsvorgang nach einer Phase der Universalisierung eines bestimmten, insbesondere von Frankreich geprägten Kulturmodells. Denken Sie nur an die Bedeutung des Französischen am preußischen Hofe oder an der Preußischen Akademie der Wissenschaften, aber auch an Ihre unmittelbare Umgebung im Park von Sanssouci und in den historischen Gebäuden am Neuen Palais! Dieser Vorgang trat folglich nach einer Phase der Entdifferenzierung unbestreitbar in eine sehr heftig verlaufende Phase erneuter Differenzierung auf nationaler beziehungsweise nationalistischer Basis ein. Ein heftiger und nachhaltiger Nationenbildungsprozess hatte überall in Europa, aber auch in vielen „Areas“ der außereuropäischen Welt eingesetzt.

Diese Herausbildungsprozesse national konstituierter Staaten bedeuteten aber keinesfalls, dass dadurch die internationalen Dimensionen in Europa oder außerhalb Europas weggebrochen wären. Im Gegenteil: Ein menschheits- und weltgeschichtliches Bewusstsein der Romantik in Hinblick auf die universelle Dimension zumindest des europäischen Kulturmodells kann schlechterdings nicht bestritten werden. Haken wir bei diesem Aspekt an zentraler Stelle in unserer ersten Annäherung an die Romantik noch einmal nach!

Wir finden diese weltgeschichtlichen Vorstellungen im Umfeld dessen, was Alexander von Humboldt das Weltbewusstsein genannt hatte, auf den verschiedensten Ebenen; eine Tatsache, die vom Auftauchen einer Reihe von Ausdrücken und Begriffen in jenen Jahren begleitet und angezeigt wird, welche stets die globale Dimension geschichtlicher Phänomene hervorkehren. Zu diesen im Umfeld der zweiten Phase beschleunigter Globalisierung entstandenen Begriffen gehören „Welthandel“ und „Weltverkehr“, „Weltwirtschaft“ und „Weltgeschichte“, aber auch „Weltbewusstsein“ und nicht zuletzt die „Weltliteratur“.

In diesem Zusammenhang erscheint es mir bedeutsam, dass gerade der große Dichter und Denker der Deutschen in einem marginalen kleinen Staatswesen, das eigentlich am Rande der damaligen Weltgeschichte lag, im mehr als beschaulichen Weimar also, als der eigentliche Präger dieses Begriffs der Weltliteratur zu sehen ist. Der entscheidende Anstoß erfolgte in den von Friedrich Nietzsche so sehr bewunderten Gesprächen mit Eckermann, in denen Johann Wolfgang von Goethe am 31. Januar 1827 zu Protokoll gab:

Aber freilich, wenn wir Deutschen nicht aus dem engen Kreise unserer eigenen Umgebung hinausblicken, so kommen wir gar zu leicht in diesen pedantischen Dünkel. Ich sehe mich daher gerne bei fremden Nationen um und rate jedem, es auch seinerseits zu tun. Nationalliteratur will jetzt nicht viel sagen, die Epoche der Weltliteratur ist an der Zeit, und jeder 
muß jetzt dazu wirken, diese Epoche zu beschleunigen. Aber auch bei solcher Schätzung des Ausländischen dürfen wir nicht bei etwas Besonderem haften bleiben und dieses für musterhaft ansehen wollen. Wir müssen nicht denken, das Chinesische wäre es, oder das Serbische, oder Calderon, oder die Nibelungen; sondern im Bedürfnis von etwas Musterhaftem müssen wir immer zu den alten Griechen zurückgehen, in deren Werken stets der schöne Mensch dargestellt ist. Alles übrige müssen wir nur historisch betrachten und das Gute, so weit es gehen will, uns daraus aneignen. ${ }^{21}$

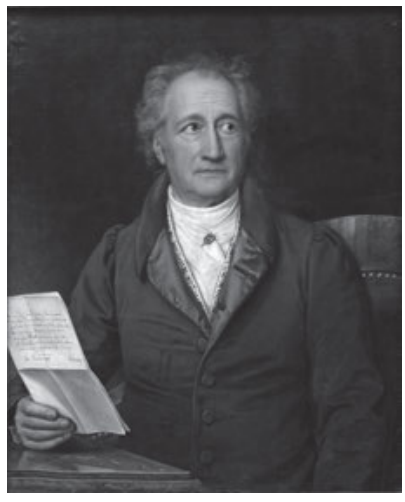

Abb. 4: Johann Wolfgang von Goethe (Frankfurt am Main, 1749 - Weimar, 1832).

Es ist aufschlussreich zu beobachten, dass sich Goethe in seinem kleinen Thüringer Provinzstädtchen literarisch noch immer als ein Weltbürger, als „citoyen de l'univers“ verstand: als Bewohner jener „République des Lettres“, welche zu Zeiten der Aufklärung bestanden hatte. Die Dimensionen dieser Republik der Literatur gehen bei ihm weit über Europa hinaus und beziehen in den Kreis der Weltliteratur ganz selbstverständlich die Literatur Chinas mit ein, so dass Goethes Begriff von „Welt“ durchaus nicht mehr nach dem restriktiven Verständnis der Aufklärung gestaltet, sondern weltumspannend gemeint war.

Der Begriff „Weltliteratur“ stellt sich einem gerade erst aufgekommenen, nach-aufklärerischen Begriff entgegen, der uns heute geradezu selbstverständlich erscheint (was er jedoch keineswegs war): dem der „Nationalliteratur“. Im Grunde ließe sich die angeführte Passage aus Eckermanns Gesprächen mit Goethe sehr wohl als Beispiel für ein Denken der Differenz ansehen, das gleichwohl wieder zurückgebogen wird auf ein Denken der Einheit. Denn es wird letztlich ausgerichtet an einem universal gültigen Meridian oder Maßstab, jenem der klassischen Antike oder genauer: dem, was die deutschen Zeitgenossen damals

21 Eckermann, Johann Peter: Gespräche mit Goethe in den letzten Jahren seines Lebens. Herausgegeben von Fritz Bergemann. Bd. I. Frankfurt a. M.: Insel Verlag 1981, S. 211: 31.1.1827. 
unter ,den Griechen' verstanden, welche sie angeblich besser als jedes andere Volk beurteilen konnten. Interessant ist dieser Denkansatz, in dem Differenz gleichsam als Motor, als Katalysator erscheint, um das Eigene voranzubringen, sehr wohl, und ich werde auf diese Vorstellungen von Weltliteratur noch mehrfach zurückkommen.

Denn der von Goethe geschaffene und von ihm selbst durchaus unterschiedlich gedeutete Begriff wird aus der Erfahrung einer Begrenztheit des Eigenen, der Erkenntnis eines Zerfallens in einzelne Nationalliteraturen entfaltet und gegen die Zuspitzung von Literatur auf das jeweils Nationale gewendet. Weltliteratur ist hierbei als Analogbildung zu Welthandel oder Weltverkehr eine Verknüpfungsmetapher, ein komplexes Verbunden-Sein, welches das Eigene (etwa das eigene Schreiben) mit dem Anderen (dem Schreiben in anderen Sprachen und Kulturen) verbindet. Dies weist selbstverständlich der Aufgabe des Übersetzers einen hohen Stellenwert zu. Denn ohne die Tätigkeit des ständigen Übersetzens zwischen verschiedenartigen Literaturen wäre die Vorstellung von Weltliteratur nichts anderes als ein sanfter Traum, was Goethe, der sich sehr für die literarische Übersetzung als Bindeglied, als „Kupplerin“ interessierte und engagierte, sehr genau wusste. 22

Vor unserem Fragehorizont ist jedoch die Einsicht in das Fragmentarische als Movens einer individuellen Vernetzung wie auch der kollektiven Beschleunigung der von Goethe so benannten „Epoche der Weltliteratur“ entscheidend. Diese Einsicht bestimmte übrigens auch Alexander von Humboldts Verständnis der Naturerfahrung in seinem Kosmos als notwendig fragmentarisches Erleben, solange dieses Erleben an ein Leben in einer Region oder Klimazone gebunden ist. Die Offenheit der Bezüge und Beziehungen im inter- und transkulturellen Zusammenspiel unterschiedlichster literarischer Texte, auf die Goethe in seiner Aussage vom 31. Januar 1827 anspielte, bedeutet keineswegs, dass es sich bei der von ihm entworfenen Weltliteratur um ein dezentriertes System handelte. Denn der Meridian seines Wertesystems verläuft durch eine aus deutscher Sicht gedeutete griechische Antike, wodurch seine Vorstellungen zweifellos europäisch zentriert erscheinen. Sein Verständnis von Weltliteratur - und bis zum heutigen Tage jedes Verständnis von Weltliteratur - besitzt ein unverkennbares Zentrum, sei dies für Goethe Weimar, für Pascale Casanova Paris oder für David Damrosch New York. ${ }^{23}$

22 Vgl. hierzu das dritte Kapitel „Translationen. Mit Worten des Anderen“ von Ette, Ottmar: ZwischenWeltenSchreiben. Literaturen ohne festen Wohnsitz (ÜberLebenswissen II). Berlin: Kulturverlag Kadmos 2005, S. 103-121.

23 Vgl. hierzu die Ausführungen in Ette, Ottmar: Von den historischen Avantgarden bis nach der 
Damit erweist sich das Wertesystem der auf den ersten Blick so weltoffenen und global ausgerichteten Vorstellung einer Weltliteratur als ein an der abendländischen Antike und Kulturentwicklung - ja mehr noch: an einer ganz bestimmten Weimarer Sichtweise der griechischen Antike - ausgerichtetes Netz von Koordinaten ungleicher Wertigkeit. Ähnlich wie die Netze europäischer Kartographen wurde es von Europa aus über die Welt ausgeworfen und legte Meridiane nach eigener Nützlichkeit fest. Damit verbinde ich selbstverständlich keine anachronistische Kritik an der abendländischen Zentriertheit bestimmter Vorstellungen, welche sich in den Weltkomposita von „Welt-Handel“ bis „Welt-Literatur“ quasi gesamtgesellschaftlich äußerten. Doch der Hintergedanke, der mich dazu bewog, Ihnen dies alles gleich zu Beginn dieser Vorlesung vorzustellen, ist natürlich die Vermutung und mehr noch die Hoffnung, dass wir heute, im dritten Jahrzehnt des 21. Jahrhunderts, in der Lage und fähig sind, ein Denken der Differenz zu entfalten. Dieses würde nicht auf einer Orientierung am Eigenen gründen und letztlich an einem homogenisierenden, europäisch zentrierten Kräftefeld ausgerichtet sein. Die Beschäftigung mit Texten des 19. Jahrhunderts verschafft uns die Möglichkeit, unsere heutige Situation historisch und kontrastiv in ihrem Geworden-Sein zu erkennen. Es gibt keinen besseren Zugang zu diesem historischen GewordenSein als die Literatur, die uns in die Lage versetzt, bewegungsgeschichtlich und aus dem Erleben selbst noch das in seiner Mobilität ästhetisch zu erkennen, was längst schon historisch geworden ist.

Wenn die Welt, wie Clifford Geertz schrieb, tatsächlich eine „Welt in Stücken“ geworden ist, dann bedeutet dies keineswegs, dass wir auf eine ethische Grundhaltung, die auf der Einheit des Menschengeschlechts basiert, verzichten müssten, wohl aber, dass es notwendig ist, diese Einheit in einem Zusammenspiel von Universalem und Partikularem, Ganzheitlichem und Fragmentarischem zu denken. Wir müssen die Welt nicht global als eine Kugel in ihrer Gesamtheit denken, um sie weltumspannend und weltweit verstehen $\mathrm{zu}$ können, sondern sollten vielmehr versuchen, sie auf eine transareale Weise gleichsam im jeweiligen Zusammenspiel einzelner „Areas“ zu begreifen. Ich werde auf diesen Grundgedanken der TransArea Studies noch wiederholt eingehen.

Dabei ist es nötig, eine relationale Logik zu entwickeln, die alles mit allem verbindet, und zwar ohne partikulare Logiken auszuschließen. Es muss also relativ autonome eigene und zugleich andersartige Logiken geben, von denen aus das Eigene und das Andere stets neu gedacht wird und als Eigenes im Anderen und als Anderes im Eigenen vorstellbar wird. Wenn wir von dieser Position aus - und

Postmoderne. Potsdamer Vorlesungen zu den Hauptwerken des 20. und 21. Jahrhunderts. Berlin Boston: Verlag Walter de Gruyter 2021. 
sie ist es, die ich im weiteren Verlauf unserer Vorlesung bewusst wählen will - die Romantik betrachten, dann können wir die Romantik in zwei Welten vielleicht auf innovative Weise so denken, dass wir sie in ihrer Differenz zusammen-denken können. Und dies soll auf eine Art geschehen, ohne sie ineinander aufzulösen oder - nicht weniger schlimm - die amerikanische Romantik als bloße Variante oder epigonale Äußerung einer europäischen Romantik zu deuten, die ihrerseits wiederum einige wenige normgebende Zentren der Ausstrahlung kennt.

Wir könnten auf diese Weise eine Dynamik zurückgewinnen, die es uns erlaubt, die fundamentale Offenheit der Zukunft in der Vergangenheit zu rekonstruieren und das Phänomen Romantik gerade nicht als nationalliterarisches oder bestenfalls europäisches, sondern als grundlegend internationalisiertes, transareales und verschiedene Kulturräume durchlaufendes Ensemble bestimmter Charakteristika zu begreifen. Dass die Spannung zwischen dem Universalen und dem Partikularen, dem Transarealen und Translokalen dabei eine wichtige Rolle zu spielen hat, dürfte inzwischen wohl klar geworden sein.

Lassen Sie mich zu diesem Fragenkomplex - vorerst abschließend - ein zusätzliches, ebenfalls von einer ethischen Grundlage her gedachtes Element hinzufügen! Denn die fundamentale Differenz und Diversität der Romantik zwischen zwei Welten - beziehungsweise der verschiedenen Romantiken - herauszuarbeiten bedeutet, damit auch eine grundlegende Dynamik und einen Reichtum der Kulturen zurückzugewinnen, welcher dieser Epoche eignet. Denn selbstverständlich erschöpft sich die Romantik nicht in Johann Gottlieb Fichtes Reden an die deutsche Nation, so wichtig diese ab Dezember 1807 gehaltenen Reden auch gewesen sein mochten. Doch greifen wir an dieser Stelle und zum Abschluss unserer ersten Annäherung an die Romantik noch einmal auf einen Anthropologen zurück!

In seinem 1952 für die UNESCO angefertigten Band Race et historie hat der französische Kulturforscher Claude Lévi-Strauss auf die nach seiner Sichtweise offensichtliche Tatsache hingewiesen, dass der Fortschritt der Menschheit - wenn überhaupt - nur als Ergebnis von Differenz und Diversität gedacht werden kann. ${ }^{24}$ Isolation, Abgeschlossenheit, Kommunikationslosigkeit verhinderten kulturellen und anderen Fortschritt auf grundlegende Weise. ${ }^{25}$ Daraus ergebe sich letztlich eine ethisch fundierte Einsicht in die Notwendigkeit kultureller Differenz, wie sie auch in Race et histoire in der unmittelbaren Zeit nach dem Zweiten Weltkrieg zum Ausdruck kam. Es handelt sich folglich um einen Zeitpunkt, zu welchem sich ein gewaltiges homogenisierendes Machtsystem in den Augen der Zeitgenossen

24 Vgl. Lévi-Strauss, Claude: Race et histoire. Paris: UNESCO 1952.

25 Ebda., S. 73. 
der Weltherrschaft zu bemächtigen anschickte und Alternativen angesichts einer raschen Aufspaltung in zwei antagonistische Blöcke weltpolitisch kaum offenstanden.

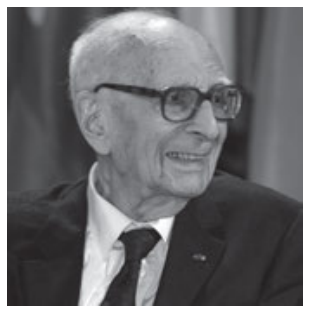

Abb. 5: Claude Lévi-Strauss (Brüssel, 1908 - Paris, 2009).

Dabei wurde bisweilen ein kultureller Isolationismus betrieben, welcher mitunter auch heute noch, in unserer Zeit nach Ende des Kalten Krieges und dem Ende der vierten Phase beschleunigter Globalisierung, immer wieder aufzuflammen droht. So schrieb der französische Strukturalist und Philosoph wenige Jahre nach dem Zweiten Weltkrieg, der das ganze Grauen von Rassismus und Antisemitismus gezeigt hatte:

Wir haben hingegen versucht zu zeigen, dass der wahre Beitrag der Kulturen nicht in einer Liste ihrer jeweils eigenen Erfindungen besteht, sondern vielmehr im differentiellen Auseinandertreten, das sich zwischen ihnen ergibt. [...]

Andererseits haben wir den Begriff einer Weltzivilisation als eine Art Grenzbegriff oder als eine verkürzte Art und Weise angesehen, einen komplexen Prozess zu signieren. Denn wenn unsere Beweisführung Gültigkeit beanspruchen darf, dann gibt es keine Weltzivilisation und kann es eine solche in einem absoluten Sinne, den man diesem Begriff oft gibt, auch nicht geben, insofern die Zivilisation die Koexistenz von Kulturen voraussetzt, welche untereinander ein Maximum an Diversität aufweisen, ja auf eine solche Koexistenz ihr Wesen gründet. Die Weltzivilisation könnte nichts anderes sein als die im weltweiten Maßstab verstandene Koalition von Kulturen, von denen eine jede ihre eigene Originalität bewahrt. ${ }^{26}$

Das ist ein starkes Plädoyer nicht für die Schöpfung und Gestaltung einer einzigen Zivilisation auf unserem Planeten, sondern für die Fortentwicklung unterschiedlicher Kulturen, deren Bedeutung für die gesamte Menschheitsgeschichte gerade in ihrer Diversität besteht. Nicht die gleichmacherische Homogenität einer einzigen Weltzivilisation kann das Ziel sein, sondern die Erhaltung aller Voraussetzungen nicht für eine Biodiversität - wie wir auf dem Gebiet der Natur sagen

26 Lévi-Strauss, Claude: Race et histoire, S. $76 \mathrm{f}$. 
würden -, sondern einer kulturellen Diversität, welche am besten für den weiteren Fortschritt des Menschengeschlechts verantwortlich zeichnet.

Versuchen wir, dieses Denken auf unsere Frage nach der Romantik zwischen zwei Welten zu übertragen! Wenn wir also die zivilisatorische Bedeutung und Leistung der Romantik einschätzen und ihren Reichtum wiedergewinnen wollen, so kommt es gerade nicht darauf an, sie auf einige wenige Grundschemata zu reduzieren und von einem oder nur sehr wenigen Zentren, die selbstverständlich in Europa liegen müssen, ausgehen zu lassen. Es geht vielmehr darum, sie als hochkomplexes System unterschiedlicher kultureller Anverwandlungen zu begreifen, das innerhalb eines tendenziell weltweiten, transarealen, durch grundlegende Asymmetrien gekennzeichneten weltliterarischen Systems stattfand. Mit diesen Asymmetrien, welche das System der Weltliteratur beziehungsweise der Literaturen der Welt bis zum heutigen Tage prägen, werden wir uns in dieser Vorlesung noch eingehend auseinandersetzen.

Denn dass Goethes Weltliteratur insgesamt ein asymmetrisches System darstellt, welches wir als unverkennbar graecozentrisch und zugleich als scharf eurozentrisch, also am Meridian der griechischen Antike und den Literaturen Europas ausgerichtet begreifen dürfen, ist nur die andere Seite der Medaille des Universalen und Weltumspannenden. Wir sollten es vor diesem Hintergrund tunlichst vermeiden, von „Abweichungen“, „Normenverletzungen“ oder „Sonderentwicklungen“ der Romantik an den Rändern Europas oder in Übersee zu sprechen. Denn es gilt vielmehr, Diversität und Differenz als Werte gerade auch kultureller Entwicklungen in den verschiedenartigsten geokulturellen Areas herauszuarbeiten, wollen wir im Laufe dieser Vorlesung auch auf die Frage nach der Bedeutung der Romantik beziehungsweise der romantischen Erfahrung für unsere heutige, in Stücke gegangene Welt kreative und differenzierte Antworten geben.

Lassen Sie mich diese erste, eher kulturtheoretische Annäherung mit den Schlussworten aus Lévi-Strauss' Race et histoire abschließen und zugleich öffnen! Es handelt sich um Überlegungen, die wie geschaffen sind für eine kritische Reflexion zum Verhältnis zwischen Toleranz und Differenz. Und dies in einer Situation, in der im heutigen Brandenburg eine rechtsradikale Partei immer gröBeren Zulauf findet und ein stetig wachsender Teil der Bevölkerung sich über die unsozialen Medien fremdenfeindlich und beleidigend äußert. Einem statischen und von oben aufrecht erhaltenen Begriff von Toleranz seien daher die Worte eines Claude Lévi-Strauss nach der Erfahrung des Zweiten Weltkriegs mahnend ins Stammbuch geschrieben:

Die Toleranz ist keine kontemplative Position, welche Nachsichtigkeiten verteilt gegenüber dem, was war, wie dem, was ist. Sie ist eine dynamische Haltung, die darin besteht, das vorherzusehen, zu verstehen und zu fördern, was sein will. Die Diversität der menschlichen 
Kulturen befindet sich hinter uns, um uns herum und vor uns. Die einzige Forderung, die wir diesbezüglich erheben könnten (und die von jedem Individuum entsprechende Pflichten abverlangt), besteht darin, dass sie sich in Formen vollzieht, von denen jede einzelne ein Beitrag zu einer gegenüber den Anderen noch größeren Großzügigkeit ist. ${ }^{27}$

Lassen wir uns folglich auf eine in jeglicher Hinsicht offene Erkundung der Romantik zwischen zwei Welten ein, die weder zwischen einer, eigenen' und einer ,fremden', weder zwischen einer ,wahren' und einer nicht mehr ganz so ,authentischen', weder zwischen einer ,normgebenden' und einer bestenfalls ,eklektischen' Romantik unterscheiden zu können glaubt! Versuchen wir vielmehr, innerhalb eines fraglos Europa-zentrischen Systems der Weltliteratur, aus der Vielgestaltigkeit die Einheit eines langen 19. Jahrhunderts zu rekonstruieren, aus der Vielverbundenheit die Zusammengehörigkeit herauszuarbeiten und zugleich die Anerkennung einer Diversität der Kulturen zu befördern, für die das Fremde zu einem gewichtigen Teil des Eigenen geworden ist, das uns befremdet, befragt und befruchtet!

27 Lévi-Strauss, Claude: Race et histoire, S. 85. 